\title{
Endoscopic Treatment for Dysplastic Barrett's Esophagus
}

\author{
Christine Kestens $\cdot$ Peter D. Siersema
}

Published online: 5 September 2014

(C) Société Internationale de Chirurgie 2014

We read the article by Subramanian et al. [1] with great interest. The authors provided a concise but complete overview of the current state of endoscopic treatment modalities for nondysplastic and dysplastic Barrett's esophagus (BE). They concluded that visible lesions in BE should be treated with endoscopic resection, either endoscopic mucosal resection (EMR) or endoscopic submucosal dissection (ESD), and with radiofrequency ablation (RFA) of residual intestinal metaplasia. We would like to make some additional comments in regard to the treatment of patients with dysplastic BE.

As mentioned in the article, current guidelines recommend endoscopic treatment, i.e., ablation, as an option for patients with low-grade dysplasia (LGD) in BE. However, before considering endoscopic treatment for a patient with LGD, it is important that the diagnosis of LGD is confirmed by an expert pathologist. It is known that histological evaluation of $\mathrm{BE}$ is associated with a high interobserver variability, especially in cases of LGD. Various studies have shown that a relatively high number of LGD cases are downstaged to nondysplastic BE after revision [2]. Furthermore, even after revision by an expert pathologist, the SURF trial reported that more than $27 \%$ of BE patients with LGD were found to have no dysplasia during follow-up [3]. This high number of patients with no dysplasia during follow-up likely can be explained by an incorrect initial diagnosis and consequently these patients probably had a much lower risk of neoplastic progression.

C. Kestens $(\square)$. P. D. Siersema

Department of Gastroenterology and Hepatology, University

Medical Center Utrecht, Heidelberglaan 100, 3584 CX Utrecht,

The Netherlands

e-mail: c.kestens@umcutrecht.nl
It is also important to emphasize that ablative treatments are not without complications, e.g., stricture formation after RFA has been reported in $7-12 \%$ of cases [3, 4]. For this reason, it is essential that further risk stratification strategies will be developed to identify the subgroup of LGD patients who will benefit from ablative treatment in order to reduce unnecessary adverse events and costs in patients without a risk of neoplastic progression. It can be expected that the inclusion of additional risk factors for developing highgrade dysplasia and adenocarcinoma in BE, e.g., a known duration of $\mathrm{BE}$ of $\geq 10$ years, longer length of $\mathrm{BE}$, and presence of esophagitis [5], may help in risk stratification. Furthermore, it is recommended to ablate LGD only after revision by an expert pathologist and with two consecutive endoscopies confirming a diagnosis of LGD.

When it is decided that a segment of Barrett's esophagus should be ablated, it is advisable that patients are referred to a center with ample experience in ablative techniques in order to improve treatment outcomes. A recent study showed that the rate of complete eradication of intestinal metaplasia after RFA increases when performed by an experienced endoscopist [6].

EMR, ESD, and RFA are all newly developed techniques and therefore long-term follow-up data are limited. Conclusions regarding the (cost-)effectiveness and recommendations on the optimal treatment strategy of different types of patients are preliminary. Furthermore, complete eradication of (dysplastic) BE does not preclude recurrence of $\mathrm{BE}$, and even after complete eradication of $\mathrm{BE}$, endoscopic surveillance is still indicated. A recent retrospective cohort study with a follow-up of up to 5 years reported a recurrence risk of 6.2 and $39.5 \%$ for dysplasia and $\mathrm{BE}$, respectively. Recurrent dysplasia was detected after a median follow-up of 44 months [7]. A small cohort study $(n=54)$ from The Netherlands reported a recurrence risk 
of $7 \%$ for dysplasia. All cases of dysplasia were detected 4-5 years after complete eradication of BE [8]. These results suggest that endoscopic surveillance after complete eradication of BE should be at least 5 years, but more data on recurrence rates and time to recurrence are required to further optimize surveillance guidelines.

In conclusion, endoscopic treatment for dysplastic $\mathrm{BE}$ has been shown to be effective in the definitive treatment of BE. In line with the article by Subramanian et al. [1], we believe that further risk stratification is necessary to select patients with LGD who will indeed benefit from endoscopic treatment. Long-term results are limited and up to now have shown that endoscopic treatment does not preclude recurrent dysplasia and/or intestinal metaplasia. Therefore, it seems advisable that patients be followed for at least 5 years after endoscopic treatment of BE.

\section{References}

1. Subramanian CR, Triadafilopoulos G (2014) Endoscopic treatments for dysplastic Barrett's esophagus: resection, ablation, what else? World J Surg. doi:10.1007/s00268-014-2636-6
2. Curvers WL, ten Kate FJ, Krishnadath KK et al (2010) Low-grade dysplasia in Barrett's esophagus: overdiagnosed and underestimated. Am J Gastroenterol 105:1523-1530

3. Phoa KN, van Vlisteren FG, Weusten BL et al (2014) Radiofrequency ablation versus endoscopic surveillance for patients with Barrett esophagus and low-grade dysplasia: a randomized clinical trial. JAMA 311:1209-1217

4. Shaheen NJ, Sharma P, Overholt BF et al (2009) Radiofrequency ablation in Barrett's esophagus with dysplasia. N Engl J Med 360:2277-2288

5. Sikkema M, Looman CW, Steyerberg EW et al (2011) Predictors for neoplastic progression in patients with Barrett's Esophagus: a prospective cohort study. Am J Gastroenterol 106:1231-1238

6. Fudman DI, Lightdale CJ, Poneros JM et al (2014) Positive correlation between endoscopist rediofrequency ablation volume and response rates in Barrett's esophagus. Gastrointest Endosc 80(1):71-77

7. Anders M, Bähr C, El-Masry MA et al (2014) Long-term recurrence of neoplasia and Barrett's epithelium after complete endoscopic resection. Gut. doi: 10.1136/gutjnl-2013-305538

8. Phoa KN, Pouw RE, van Visteren FGI et al (2013) Radiofrequency ablation with endoscopic resection: a Netherlands cohort study. Gastroenterology 145:96-104 